\title{
Accuracy and Feasibility of Point of Care (POC) Early Infant Diagnosis (EID) in Nigeria: A Field Evaluation
}

\author{
Busari Olusegun \\ Department of Public Health, Texila American University, Guyana
}

\begin{abstract}
The timely commencement of treatment within the first 12 weeks of life in children reduces the HIV disease progression by $75 \%$ and mortality by $76 \%$. For all children infected with HIV to receive essential treatment and care, few things are more important than early and accurate HIV diagnosis. Traditional methods of providing Early Infant Diagnosis (EID) have been bedeviled with several challenges. Point of Care (POC) testing for EID has been recommended as a game-changer. A field evaluation was conducted across 6 healthcare facilities between February 2017 and August 2018 to determine the accuracy and operational feasibility of POC testing for EID in Nigeria. The conceptual framework rides on the WHO SDI ASSURED criteria. Participants were infants aged between 4 weeks and 18 months born to HIV-positive mothers and visiting the clinic for the first time for EID. Results obtained from the POC was compared with the Standard of Care (SOC) to determine accuracy and feasibility. A total of 245 participants took part in the study, with $76 \%$ visiting the clinic for EID within 2 months of life. The average turnaround time for EID test using the SOC was 67 days. Analysis of the $10.4 \%$ error rate indicate significant correlation by personnel $(r=0.01122, P=0.841706)$. An average concordance percentage of $99.6 \%$, sensitivity of $91.7 \%$ and specificity of $100.0 \%$ was observed. Operator's assessment indicates that the equipment is very good and acceptable. The POC testing for EID is acceptable, rapid, and robust; hence, viable for use in Nigeria.
\end{abstract}

Keywords: Early Infant Diagnosis, HIV/AIDS, Point of Care.

\section{Introduction}

Early diagnosis of HIV and the commencement of HIV-infected infants on ART within the first 12 weeks of life can save a significant number of lives [1]. Without access to treatment, which is determined by early diagnosis, about one-third of HIV-infected infants will die by age 1 year, and $50 \%$ by age 2 years [2]. Evidence has shown that the timely commencement of treatment in children reduces the disease progression by $75 \%$ and mortality from the infection by $76 \%$. However, only $54 \%$ of children living with HIV globally are on lifesaving antiretroviral therapy. In the sub-Saharan Africa region, which accounts for $91 \%$ of the 1.7 million children living with HIV/AIDS globally, ART coverage in children is 52\%: with the West and Central Africa region having the lowest regional ART coverage globally-28.4\% [3].

For all children infected with HIV to receive essential treatment and care, few things are more important than early and accurate HIV diagnosis. Early Infant Diagnosis (EID) aids the early detection of HIV infection in children, detection of prenatal, intrapartum, and postnatal transmission of HIV infection, timely initiation and commencement of life-saving antiretroviral therapy for HIV positive infants; as well as identifying successful prevention of mother to child transmission of HIV in HIV negative children [4].

Despite the many benefits of Early Infant Diagnosis, its uptake has been bedeviled with several challenges; resulting in high attrition and loss to follow up among the mother-baby pair among others [5]. Notwithstanding the many innovative solutions to address these challenges; the proportion of HIV exposed infants (HEI) who had virological testing to assess HIV status remains low. As of 2018, globally only $58.7 \%$ of infants born to pregnant women living with HIV had a virological test for HIV within 2 months of birth; with the West and Central Africa regions having an abysmal coverage of $29.3 \%$ [3].

According to the UNAIDS 2019 report, $46.4 \%$ of new infections (Adults and Children) in the West and Central Africa region occurred 
in Nigeria. In children (0-14years), a total of 24,000 new infections and 14,000 AIDS-related deaths were recorded in Nigeria. This is the highest number of new infection and AIDSrelated death in children globally. However, only $23.8 \%$ of HIV-exposed infants received an EID test and $35 \%$ of children living with HIV received treatment in 20183. In Nigeria, virological testing for Early Infant Diagnosis is provided through centralized PCR laboratories and relying on the sample referral network for the transportation of samples and return of results. Studies have shown the earliest return of results from the centralized laboratory to be at 3 months and 5.6 months, even with $81.7 \%$ and $62.3 \%$ collection of DBS samples within the stipulated 2 months by Nigeria National guideline [5,6]. Documented challenges with EID are similar to other sub-Saharan countries including stock-out of DBS commodities, collection of DBS samples only on a few designated days long TAT, delayed result return leading to loss to follow up (LTFU) and suboptimal sample referral network efficiency among others $[7,8]$.

Three (3) innovative approaches an early infant testing, expanded postnatal prophylaxis (ePNP); and Point of Care nucleic acid testinghas been recommended by the World Health Organization (WHO) as game changers in achieving prompt diagnosis of HIV in infants and initiation on treatment. The use of Point of Care (POC) nucleic acid testing has been shown to significantly increase access and uptake of EID by providing the platform for HIV nucleic acid testing at the point of service delivery, outside of the centralized laboratories. Also, notable advantages which impact patient outcomes have been alluded to POC technologies $[9,10]$. Currently, while expanded, postnatal prophylaxis (ePNP) are provided for HIV exposed infants; the use of point of care (POC) technologies for providing early infant diagnosis is yet to be introduced in Nigeria; despite several POC EID equipment evaluated and in use across some countries in sub-Saharan Africa. The Alere ${ }^{\mathrm{TM}} \mathrm{q}$ platform employs the Alere $^{\mathrm{TM}}$ q HIV1/2 - Detect, which is a qualitative method using the nucleic acid-based test for Early Infant Diagnosis. This has been prequalified by the WHO in 2016 as a POC for
Early Infant Diagnosis of HIV and is in use across some sub-Saharan Africa countries [11]. With the main goal of addressing gaps in ensuring early and reliable diagnosis of HIV in infants and expand access to treatment, which is a critical consideration for HIV epidemic control in Nigeria; this study assessed the accuracy and operational feasibility of using a point of care testing for Early Infant Diagnosis in Nigeria.

\section{Materials and Methods}

\section{Study Setting}

The study was conducted across six (6) healthcare facilities in three (3) states-Kaduna, Kogi, and Benue-located within the north-west and north-central geopolitical zone of Nigeria states. The healthcare facilities cut across urban, peri-urban, and rural areas; as well as the three (3) levels of the health systems in Nigeria Primary, Secondary, and Tertiary healthcare levels. Nigeria has a high burden of HIV/AIDS, second only to South Africa by global ranking; with an HIV prevalence of $1.4 \%$ and an estimated 1,900,000 persons living with HIV.

\section{Study Population}

The study population was infants born to HIV-positive mothers aged between 4 weeks and 18 months and visiting the clinic for the first time for the virological Early Infant Diagnosis test between February 2017 and August 2018.

\section{Conceptual Framework}

Employing a mixed-method approach, the conceptual framework (see Figure 1) rides on the World Health Organization (WHO) Sexually Transmitted Diseases Diagnostics Initiative (SDI) ASSURED (Affordable, Sensitive, Specific, User-friendly, Rapid and robust, Equipment-free and Deliverable to end-users) criteria as a benchmark for point-of-care (POC) tests which are recommended for resource limited settings [12]. Of the 7 WHO SDI ASSURED criteria, 5 were assessed through a quantitative comparative analysis of the POC with the gold standard/ local SOC. The assessment of the other 2 criteria involved the use of a qualitative analysis leveraging standardized questionnaires to abstract users' perspectives. 


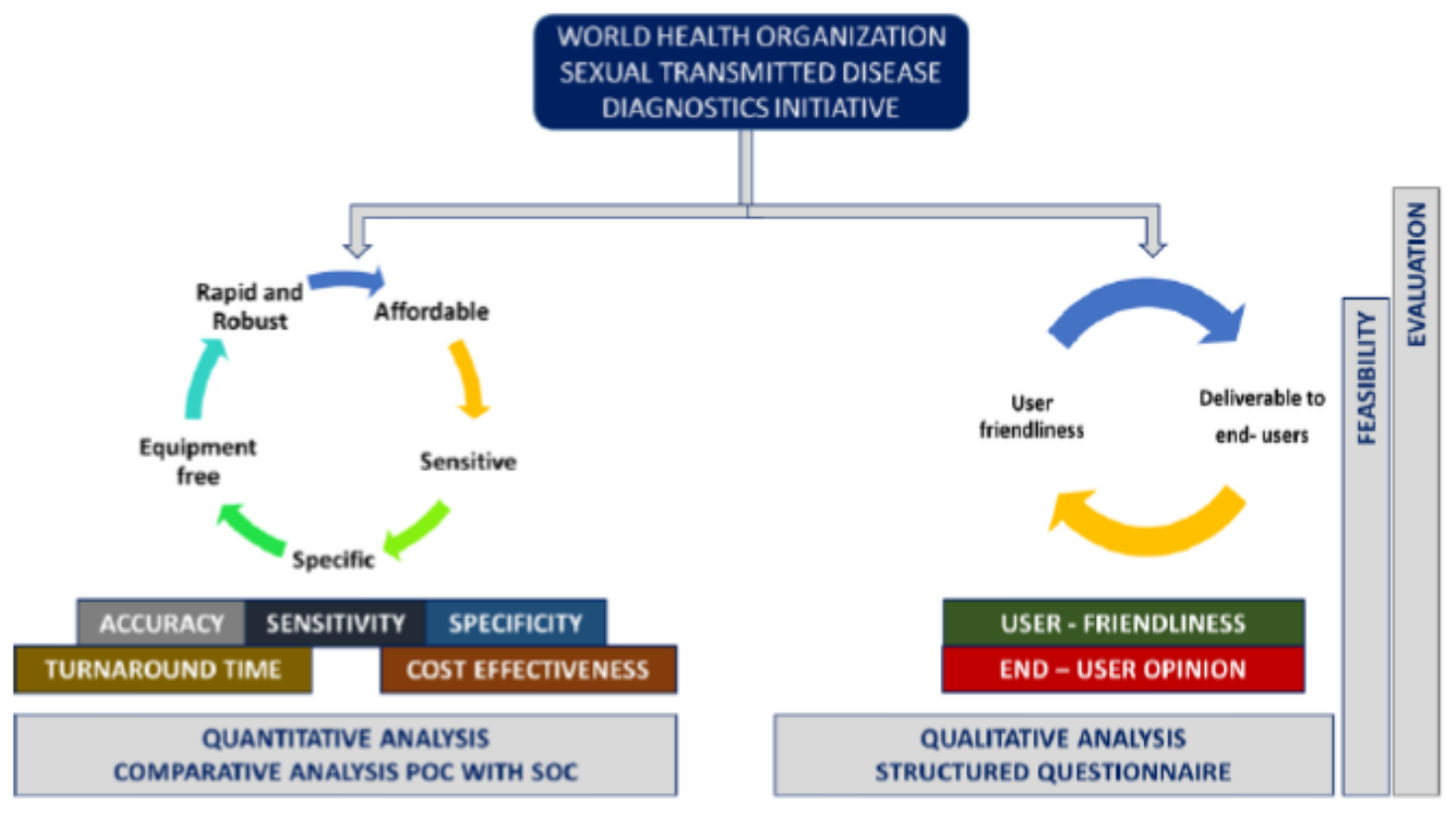

Figure 1. Conceptual Framework Based on WHO SDI Assured Criteria

\section{Study Design}

The study adopted a cross-sectional study design to evaluate the accuracy and feasibility of using the Point of Care (POC) N - Alere ${ }^{\mathrm{TM}} \mathrm{q}$ HIV-1/2 Detect - for Early Infant Diagnosis among HIV Exposed Infants across 2 tertiary, 3 secondary and 1 primary level healthcare facilities in Nigeria. All eligible and consenting participants were recruited consecutively across the states between February 2017 and August 2018. The selection of healthcare facilities was based on 1-year retrospective data on infant attendance. Written or thumb printed parental/guardian consent was requested for all infants to be enrolled. No infant was excluded based on gender, socioeconomic, racial, or ethnic grounds. Demographic information including gender and birth date for study participants were documented.

\section{Sample Size}

Sample size was calculated using the formula:

Where in,

$$
\text { Sample size, } \mathbf{n}=\mathrm{N} * \frac{\frac{Z^{2} * p *(1-p)}{e^{2}}}{\left[N-1+\frac{Z^{2} * p *(1-p)}{e^{2}}\right]}
$$

N, Population Size - 98,413.

(Total number of Infants (0-18 months born to HIV positive mothers in 2016).

$\mathrm{P}$, Population proportion $-18.9 \%$.
(Proportion of infants ( $0-18$ months) who had access to virological testing in 2016).

$\mathrm{Z}$, Critical Value at 95\% Confidence level 1.96.

e, Margin of error - $5 \%$.

The calculated Sample size is 235 .

\section{Sampling Technique}

Health facilities were selected across the 3 states based on 1-year historical volume of HIV Exposed Infants accessing virological testing. Consecutive sampling method was used in recruiting clients meeting the inclusion criteria for the evaluation study across 6 selected healthcare facilities. Written or thumb printed parental/guardian consent were requested for all infants to be enrolled. No infant was excluded on the basis of gender, socioeconomic, racial, or ethnic grounds. Demographic information including gender and birth date for study participants were documented.

\section{Procedure}

Group consent sessions were held for all clients visiting the clinic. This was followed by individual consent sessions where the criteria of participants to be part of the study are confirmed and individual consents documented. From each study participant, Dried Blood Spot (DBS) was collected for Early Infant Diagnosis (EID) using the Standard of Care (SOC). Six (6) blood spots were taken per each study participant and 
Quality Control verification carried out to ensure proper collection. During sample collection, $25 \mu \mathrm{l}$ of blood was collected and analyzed for Early Infant Diagnosis using the Alere ${ }^{\mathrm{TM}} \mathrm{q}$ HIV 1/2 Detect at the Point of Care (POC). Across each of the selected facilities, qualified healthcare workers who routinely collect Dried Blood Spot (DBS) were responsible for DBS sample collection and Alere ${ }^{\mathrm{TM}} \mathrm{q}$ HIV-1/2 Detect test. All healthcare workers responsible for DBS sample collection and assigned for the POC test were trained on the conduct of the Alere ${ }^{\mathrm{TM}} \mathrm{q}$ HIV-1/2 Detect test for EID. Error codes and other instrument error logs were documented by operators to establish the overall error rate. Results from the Alere ${ }^{\mathrm{TM}} \mathrm{q}$ HIV-1/2 Detect were documented. In line with the SOC, DBS was transported to the centralized PCR laboratories for analysis. At the centralized PCR laboratories, all DBS were assayed using the COBAS ${ }^{\circledR}$ AmpliPrep/COBAS ${ }^{\circledR} \quad$ TaqMan ${ }^{\circledR} \quad$ HIV-1 Qualitative Test, version 2.0; and results returned to the healthcare facility. Critical information to measure the turnaround time (TAT) and document test records aligned to the unique study identification number were captured and kept separately for each of POC and SOC to ensure participants' confidentiality and anonymity and avoid bias. Additionally, structured questionnaires were administered to healthcare workers including operators of the POC; across healthcare facilities to assess their views and opinion on the POC for EID. The operators assessed the equipment based on Turnaround Time (TAT), Quality of results, Reliability, Accuracy, Operation, Safety, and User-friendliness. Each of the criteria was graded by the operators and assessment points were weighted to determine final graded performance.

\section{Data Management and Analysis}

For the quantitative methods, participant's demographic data were abstracted using the participant enrollment forms. Study tools were developed and used to capture the study of technical data, including all process data as well as the outcome. Also, results from the Point of Care equipment were transmitted electronically through the Alere data point. This was accessed remotely electronically and monitored to ensure adherence to study protocol. Information captured on the Alere data point and all study tools were triangulated to validate the correctness and assure data integrity before analysis. Descriptive analysis was carried out to summarize the outcome measures and the background characteristics of all study participants using percentages for categorical variables and mean, median and standard deviations for quantitative variables. The accuracy (bias and limits of agreement) of the Alere $^{\mathrm{TM}}$ q HIV 1/2 - Detect in comparison with the COBAS ${ }^{\circledR}$ AmpliPrep/COBAS $®$ TaqMan ${ }^{\circledR}$ HIV-1 Qualitative Test, version 2.0 was determined. These include sensitivity, specificity, positive and negative predictive values. All Analyses will be carried out using Stata Version 14 (StataCorp, College Station, Texas, USA); and statistical level of significance set at $\mathrm{P}$-value $\leq 0.05$

\section{Ethical Considerations}

Ethical approvals were obtained from the Kaduna, Benue, and Kogi States Ministry of Health Research and Ethics Review Committee. Written informed consent was obtained for entry into the evaluation study from their guardian/parent of the infants. The evaluation study was described, and consent requested in the local language or English. Study participants unable to sign due to illiteracy use their thumbprint as a signature.

In carrying out the study, three (3) measure were put in place to assure privacy and confidentiality of participants. These are the use of assigned unique study identification number per participants, restricted access to study data with administrative permission level for all electronic and cloud-based data; and separation of study documentation from clinical data; triangulation of data only took place during analysis.

\section{Results}

The goal of this study is to assess the accuracy and feasibility of Alere ${ }^{\mathrm{TM}} \mathrm{q}$ point-of-care (POC) nucleic acid-based test (NAT) for early infant diagnosis in Nigeria. 


\section{Distribution of Healthcare Facilities and Socio-demographic Characteristics of Participants}

This study was conducted across six (6) healthcare facilities in 3 states located within the north-west and north-central region of Nigeria for infants aged 4 weeks-18 months with consenting parent/guardian. The healthcare facilities-2 Tertiary healthcare facilities, 3 Secondary healthcare facility and 1 primary healthcare facility; are located in the urban and peri-urban area of the states. The average distance from the healthcare facilities to the nearest centralized PCR laboratory is $204.8 \mathrm{~km}$ ranging from $81.7 \mathrm{~km}-371.3 \mathrm{~km}$ (see Table 1). A total of 249 participants took part in the study within the 19 months, 278 tests were conducted using the POC including 29 repeat tests. Four (4) participant's SOC results were yet to be returned at the end of the study, hence, were not included in the analysis. Out of all the study participants, $135(55 \%)$ were male and $110(45 \%)$ were female. Based on the age-group, $76.3 \%$ of the participants are $\leq 8$ weeks old, $17.1 \%$ participants are within the age group 9 weeks-12 months, while $6.5 \%$ are within the age-group $>12$ - 18 months (see Table 2).

Table 1. Distribution of Healthcare Facilities, Type, Location and Distance form PCR Laboratory

\begin{tabular}{|l|l|l|l|l|}
\hline \multirow{2}{*}{ State } & Facility & Type & Location & $\begin{array}{l}\text { Distance from the } \\
\text { assigned PCR } \\
\text { Laboratory }\end{array}$ \\
\hline \multirow{2}{*}{ Benue } & General Hospital Sankera & Secondary HF & Peri-urban & $201 \mathrm{~km}$ \\
\cline { 2 - 5 } & CHC, Gboko & Primary HF & Urban & $92.3 \mathrm{~km}$ \\
\hline Kaduna & $\begin{array}{l}\text { Barau Dikko Teaching } \\
\text { Hospital }\end{array}$ & Tertiary HF & Urban & $81.7 \mathrm{~km}$ \\
\cline { 2 - 5 } & General Hospital, Kafanchan & Secondary HF & Urban & $281.5 \mathrm{~km}$ \\
\hline \multirow{2}{*}{ Kogi } & $\begin{array}{l}\text { Federal Medical Centre, } \\
\text { Lokoja }\end{array}$ & Tertiary HF & Urban & $201 \mathrm{~km}$ \\
\cline { 2 - 5 } & General hospital Ankpa & Secondary HF & Peri-urban & $371.3 \mathrm{~km}$ \\
\hline
\end{tabular}

Table 2. Distribution of Study Participants Based on Age and Gender

\begin{tabular}{|c|c|c|c|}
\hline \multirow{3}{*}{ Age - group } & \multirow{3}{*}{ Total no. of Infants (N \%) } & \multicolumn{2}{|l|}{ Sex } \\
\hline & & $\mathbf{M}$ & $\mathbf{F}$ \\
\hline & & $(\mathrm{N} \%)$ & $(\mathbf{N} \%)$ \\
\hline$\leq 8$ weeks & $187(76.3 \%)$ & $99(53 \%)$ & $88(47 \%)$ \\
\hline 9 weeks - 12 months & $42(17.1 \%)$ & $26(62 \%)$ & $16(38 \%)$ \\
\hline$>12-18$ months & $16(6.5 \%)$ & $10(63 \%)$ & $6(37 \%)$ \\
\hline All & $245(100.0 \%)$ & $135(55 \%)$ & $110(45 \%)$ \\
\hline
\end{tabular}

\section{Facility Level Turnaround Time (TAT) for Early Infant Diagnosis (EID) Using the Standard of Care}

As part of the comparison and assessing the impact and need for the POC, the turnaround time for the return of results was captured. Turnaround time (TAT) was defined from the time of collection of the DBS samples for EID till the time the result was received from the centralized laboratory (SOC) at the health facility. The facility level turnaround time for EID test using the SOC ranges between 51 to 99 days (see Figure 2). As per age-group, the TAT ranges between 67 days for infants $\leq 8$ weeks, 50 days for infants 9 weeks -12 months and 62 days for infants within the age range $>12-18$ months. The average turnaround time for EID test using the SOC (Centralized PCR laboratories) is 67 days for infants visiting the clinic within 2 months after birth, as well as for all infants participating in the study (see Table 3). The TAT for the POC is 0 day as all results were obtained within 1-2 hours. 


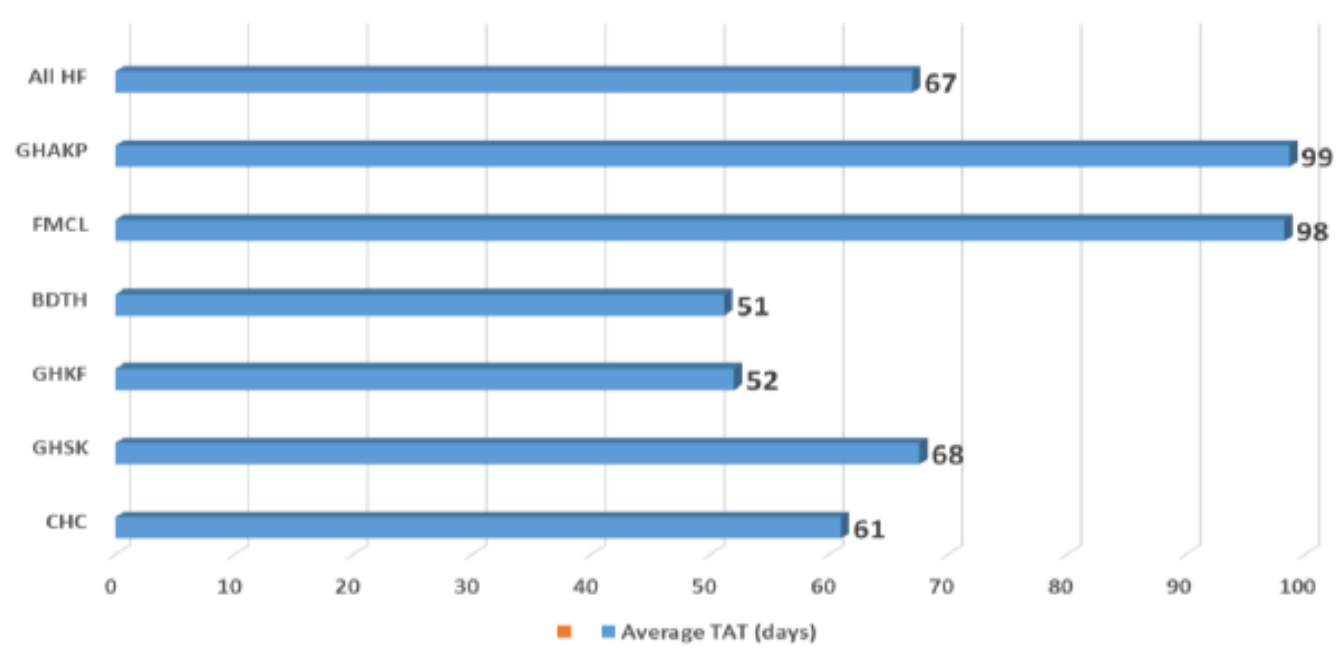

Figure 2. Facility Level Turnaround Time (TAT) on the Standard of Care (SOC)

Table 3. Turnaround Time (TAT) Per Age-Group Based on the Standard of Care (SOC)

\begin{tabular}{|l|l|l|l|l|}
\hline \multirow{2}{*}{ Age - group } & \multirow{2}{*}{ Total no. of Infants } & \multicolumn{3}{l|}{ Turnaround Time (TAT) } \\
\cline { 3 - 5 } & & Avg. TAT (days) & Range (days) & $\begin{array}{l}\text { Avg. TAT @ } \\
\text { HF (days) }\end{array}$ \\
\hline$\leq 8$ weeks & 187 & 67 & $17-138$ & 5 \\
\hline 9 weeks -12 months & 42 & 50 & $17-135$ & 5 \\
\hline$>12-18$ months & 16 & 80 & $39-121$ & 5 \\
\hline All & 245 & 67 & $14-144$ & 5 \\
\hline
\end{tabular}

\section{Analysis of Error Rate on the Point of Care (POC) - AlereTM q Platform}

In this study, a total of 278 Alere $^{\mathrm{TM}} \mathrm{q}$ HIV $1 / 2$ - Detect nucleic acid-based tests were conducted throughout the 19 months' period; including 29 repeat tests due to errors. Hence, $10.4 \%$ error rate. The error rate per facility ranges from $2.3 \%$ to $18.2 \%$ (see Table 4). All repeated tests came out with valid results. All errors recorded were analyzed based on the error code. Analysis indicates human error mostly due to low sample volume and partial entry of the cartridge into the equipment. In this study, 2 types of healthcare workers-Nurses and Laboratory personnel were responsible for carrying out the tests on the Alere $^{\mathrm{TM}} \mathrm{q}$ platforms. A regression analysis of the error rate based on the type of personnel handling the equipment indicate a significant adjusted regression of $0.01122(\mathrm{P}=0.841706)$.

Table 4. Analysis of Error Rate on Point of Care - AlereTM q Platform

\begin{tabular}{|l|l|l|l|l|l|}
\hline Facility & Personnel & Location & $\begin{array}{l}\text { Number of } \\
\text { Sample Run }\end{array}$ & $\begin{array}{l}\text { No of error } \\
\text { recorded }\end{array}$ & Error Rate \\
\hline CHC Gboko & Nurse & Urban & 84 & 12 & $14.3 \%$ \\
\hline GH Sankera & Nurse & Peri-urban & 55 & 10 & $18.2 \%$ \\
\hline BDTH & Nurse & Urban & 41 & 3 & $7.3 \%$ \\
\hline $\begin{array}{l}\text { GH } \\
\text { Kafanchan }\end{array}$ & Laboratory & Urban & 40 & 2 & $5.0 \%$ \\
\hline FMC Lokoja & Laboratory & Urban & 15 & 1 & $6.7 \%$ \\
\hline ZH Ankpa & Laboratory & Peri-urban & 43 & 1 & $2.3 \%$ \\
\hline TOTAL & 278 & 29 & $10.4 \%$ \\
\hline
\end{tabular}


Adjusted $\mathrm{R}^{2}-0.618513, \mathrm{P}=0.063533$ (-0.18469, 0.007974)

\section{Accuracy, Positive Predictive Value, and Negative Predictive Value of the Point of Care (POC) in Comparison with Standard of Care (SOC) for Early Infant Diagnosis}

A comparison of results recorded from the POC, Alere ${ }^{\mathrm{TM}} \mathrm{q}$; and the SOC, Roche Cobas CAP/CTM 48/96 indicate a non-concordance in only 1 of the entire 245 study participant's valid test conducted. An average concordance percentage of $99.6 \%$ with ranges between $98.6 \%$ and $100 \%$ across the 6 healthcare facilities was observed in this study (see Table 5). Outcome reveals sensitivity and specificity of $91.7 \%$ and
Adjusted $\mathrm{R}^{2}-0.01122, \mathrm{P}=0.841706(-0.135$, $0.157441)$

$100 \%$ respectively. A repeat of the discordant test by both platforms-SOC and POC-increased the sensitivity to $100 \%$. The POC was found to be consistent while the initial result provided by the SOC was found to be false positive. The sensitivity increased to $100 \%$ after the repeat testing of both SOC and POC. In this study, a Positive Predictive Value (PPV) of 100.0 and Negative predictive value of 99.6 was recorded. Accuracy of the POC nucleic acid-based test (NAT) in comparison with the Standard of Care in detecting HIV infection among infants aged 4 weeks to 18 months is $99.6 \%$.

Table 5. Sensitivity, Specificity and Accuracy of the POC in Relation with the SOC

\begin{tabular}{|c|c|c|c|c|c|c|}
\hline \multirow[b]{2}{*}{ Facility } & \multirow{2}{*}{$\begin{array}{l}\text { Number of } \\
\text { Sample Run }\end{array}$} & \multicolumn{2}{|c|}{ POC (Alere ${ }^{\mathrm{TM}} q$ ) } & \multicolumn{2}{|c|}{ SOC (Cobas CAP/CTM) } & \multirow[b]{2}{*}{$\begin{array}{l}\text { Concordance } \\
(\%)\end{array}$} \\
\hline & & $\begin{array}{l}\text { Total } \\
\text { Positive }\end{array}$ & $\begin{array}{l}\text { Total } \\
\text { Negative }\end{array}$ & $\begin{array}{l}\text { Total } \\
\text { Positive }\end{array}$ & $\begin{array}{l}\text { Total } \\
\text { Negative }\end{array}$ & \\
\hline CHC Gboko & 72 & 0 & 72 & 1 & 71 & 98.6 \\
\hline GH Sankera & 45 & 0 & 45 & 0 & 45 & 100.0 \\
\hline BDTH & 38 & 1 & 37 & 1 & 37 & 100.0 \\
\hline GH Kafanchan & 38 & 4 & 34 & 4 & 34 & 100.0 \\
\hline FMC Lokoja & 11 & 2 & 9 & 2 & 9 & 100.0 \\
\hline ZH Ankpa & 41 & 4 & 37 & 4 & 37 & 100.0 \\
\hline Total & 245 & 11 & 234 & 12 & 233 & 99.6 \\
\hline
\end{tabular}

Sensitivity $-91.7 \%, 95 \mathrm{CI}(0.9116,92167)$, Specificity $-100.0 \%, 95 \mathrm{CI}(1.0000,1.0000)$.

PPV - 100.0, 95CI (1.0000, 1.0000), NPV - 99.6, 95CI (0.99546, 0.99599).

Accuracy $-99.6 \%$.

\section{Operator's Appraisal of the Performance of Point of Care (POC) in Early Infant Diagnosis (EID) Testing}

Assessment of the POC by the healthcare workers responsible for the operation of the equipment' during the study covers defined performance criteria of Turnaround Time
(TAT), Quality of results, Reliability, Accuracy, Operation, Safety, and User-friendliness. The assessment of the POC equipment and test across the different criteria shows a weighted average ranging from $63 \%$ to $83 \%$ (see Table 6). Based on the graded weighted average, operators found the equipment very good across all the key criteria assessed.

Table 6. Operator's Assessment of POC Based on Defined Performance Criteria

\begin{tabular}{|c|c|c|c|c|c|c|c|}
\hline \multirow[b]{2}{*}{$\begin{array}{l}\text { Performance } \\
\text { Criteria }\end{array}$} & \multicolumn{5}{|c|}{ Grading } & \multirow{2}{*}{$\begin{array}{l}\text { Weighted } \\
\text { Average } \\
(\%)\end{array}$} & \multirow[b]{2}{*}{$\begin{array}{l}\text { Graded } \\
\text { Performance }\end{array}$} \\
\hline & $\begin{array}{l}\text { Poor } \\
\text { (0) }(\%)\end{array}$ & $\begin{array}{l}\text { Fair } \\
\text { (1) }(\%)\end{array}$ & $\begin{array}{l}\text { Satisfactory } \\
\text { (2) }(\%)\end{array}$ & $\begin{array}{l}\text { Very Good } \\
\text { (3) }(\%)\end{array}$ & $\begin{array}{l}\text { Excellent } \\
\text { (4) }(\%)\end{array}$ & & \\
\hline Turnaround Time & 0.0 & 16.7 & 0.0 & 50.0 & 33.3 & 75 & (3) \\
\hline Quality of Result & 16.7 & 0.0 & 0.0 & 0.0 & 83.3 & 83 & (3) \\
\hline Reliability & 16.7 & 0.0 & 0.0 & 66.7 & 33.3 & 67 & (3) \\
\hline Accuracy & 16.7 & 0.0 & 0.0 & 0.0 & 83.3 & 83 & (3) \\
\hline Operation & 0.0 & 16.7 & 33.3 & 33.3 & 16.7 & 63 & (3) \\
\hline Safety & 16.7 & 0.0 & 16.7 & 50.0 & 16.7 & 63 & (3) \\
\hline User-friendliness & 0.0 & 0.0 & 50.0 & 50.0 & 0.0 & 63 & (3) \\
\hline
\end{tabular}




\section{Discussion}

The study was conducted with the aim of assessing the accuracy and operational feasibility of using a point of care testing for Early Infant Diagnosis in Nigeria. Insight into this was expected to aid in addressing gaps in ensuring early and reliable diagnosis of HIV in infants and expand access to treatment, which is a critical consideration for HIV epidemic control in Nigeria. This study was conducted among infants aged 4 weeks -18 months with consenting parent/guardian across six (6) healthcare facilities in 3 states of Kaduna, Kogi and Benue states. A critical issue that has being identified with the provision of Early Infant Diagnosis (EID) service in Nigeria is distance from the centralized PCR laboratory. In this study, the distance of the healthcare facilities from the centralized PCR laboratories ranges between $81.7 \mathrm{~km}-371.3 \mathrm{~km}$; with an average distance of $204.8 \mathrm{~km}$.

According to the recent WHO guideline, Early Infant Diagnosis is recommended to be conducted within 4-6 weeks of life however, the Nigeria National guideline for HIV/AIDS Prevention, Treatment and Care recommends 2 months (8 weeks) of life. According to the findings from this study, $41 \%$ of the infants visited the clinic for early infant diagnosis for the first time at age 4-6 weeks, while $76 \%$ of the study participants visited the clinic for Early Infant Diagnosis within 2 months after birth. This suggests that majority of children still visit the clinic for early infant diagnosis within the time period that ART commencement will aid optimal survival rate. The $72 \%$ DBS collection within 2 months of life is higher than the $62.3 \%$ finding of [13] in South-Eastern Nigeria and $50.1 \%$ of [14] in North central Nigeria; however, this correlates with the $81.1 \%$ observed in the Moment study in Nigeria as reported by [6] Also, $17 \%$ of the study participants visited the clinic between 9 weeks-12 months after birth and $7 \%$ of the infants visited after 12 months to 18 months after birth. Notwithstanding the percentage of infants having access to EID within the recommended period; this study indicates a gap of $24 \%$.

One of the issues that has been identified with the optimal uptake of EID, especially in resource limited countries is the long TAT. This has been shown to delay the commencement of HIVinfected Infants on treatment and Hence, adversely affects the survival of these infants. The Moment study in north central Nigeria and the Healthy Beginning Initiative in south eastern Nigeria, has indicated a TAT of 3 months and 5.6 months respectively. In this study, the average turnaround time for EID test using the SOC (Centralized PCR laboratories) is 67 days for infants visiting the clinic within 2 months after birth, as well as for all infants participating in the study. The TAT for the POC is 0 day as all results were obtained within 1-2 hours. Average time the EID samples spent at each of the healthcare facility is 5 days. Although lesser than other studies, however, this establishes the continuous challenge with long TAT for the receipt of results from the centralized PCR laboratories; and hence, delayed commencement of ART in infants.

An analysis of sample analysis with the Point of Care-Alere ${ }^{\mathrm{TM}} \mathrm{q}$ HIV-1/2 Detect, indicate an error rate of $10.4 \%$. This is comparable to the 9.0\% recorded in the study assessing Alere ${ }^{\mathrm{TM}} \mathrm{q}$ POC for EID in South Africa by [10] as well as the $11.2 \%$ error rate observed in Zimbabwe by [15]. However, in the study in Zimbabwe, the error rate decreases with time due to familiarity with the use of equipment and increased competency. This underscores the need for continuous training to assure competency and reduce error rat. In this study, all error tests were repeated with valid results. The error rate observed in this study is far lesser than the error rate reported in the CE-IVD and WHO prequalification. This may be attributed to the fact that prequalification and certification were laboratory based. An analysis of the error rate based on the type of personnel handling the equipment indicate a significant adjusted regression of $0.01122 \quad(\mathrm{P}=0.841706)$. This suggests that the type of personnel handling the equipment has an impact on the frequency of error rate recorded. However, [15] in the study carried out in Zimbabwe did not find any a significant error rate between laboratory and non-laboratory personnel. Error rate based on location which is either urban or peri-urban was found not significant.

With an average concordance of $99.6 \%$ between the Point of Care (POC) and the Standard of Care (SOC), the results obtained with the two platforms shows a comparable accuracy. The key focus of this study is to assess the accuracy and feasibility of the Point of care 
for Early Infant Diagnosis in Nigeria. In comparison with the SOC, the sensitivity and specificity obtained in this study was $91.7 \%$ and $100.0 \%$ respectively; with a positive predictive value of 100.0 and negative predictive value of 99.6. In the evaluation carried out in Cape Town South Africa, [10] observed $95.5 \%$ sensitivity and $99.8 \%$ specificity. While the sensitivity is higher than the one observed in this study, the specificity is well comparable. Also, a repeat of the only discordant test in this study by both platforms-SOC and POC-increased the sensitivity to $100 \%$. The POC was found to be consistent while the initial result provided by the SOC was found to be false positive. Results obtained shows that the Point of Care platformAlere $^{\mathrm{TM}} \mathrm{q}$ platform is reliable and has comparative ability with the standard of care for early infant diagnosis. Also, operator's assessment of performance criteria found the equipment very good and acceptable, with

\section{References}

[1] AVERT: Global HIV and AIDS Statistics. (February, 2020). Date of Access: 5/04/2020 https://www.avert.org/global-hiv-and-aidsstatisticsUpdated.

[2] WORLD HEALTH ORGANIZATION (WHO). Global Programme on AIDS, 1988. WHO report Breastfeeding, breast milk and human immunodeficiency virus (HIV) Statement from the Consultation held in Geneva, 23-25 June, 1987: AIDS Action, (5), 1-2: Date of Access: 05/05/2020. https://pubmed.ncbi.nlm.nih.gov/12281628/.

[3] UNAIDS Data 2019. Date of Access: 06/05/2020 https://www.unaids.org/sites/default/files/media_ass et/2019-UNAIDS-data_en.pdf.

[4] Anisa Ghadrshenas, Yanis B. Amor, Joy Chang, Helen Dale. et al. (2013). Improved access to early infant diagnosis is a critical part of a child-centric prevention of mother-to-child transmission agenda. AIDS 2013, 27 (Suppl. 2): S197-S205. Date of Access: $15 / 5 / 2020$

https://journals.lww.com/aidsonline/Fulltext/2013/1 1002/Improved_access_to_early_infant_diagnosis_i s_a.8.aspx.

[5] Carlucci, J. G., Liu, Y., Friedman, H., Pelayo, B. E., Robelin, K., Sheldon, E. K., Clouse, K., \& Vermund, S. H. 2018. Attrition of HIV-exposed infants from early infant diagnosis services in lowand middle-income countries: a systematic review and meta-analysis. Journal of the International AIDS weighted rating average ranging from $63 \%$ to $83 \%$.

\section{Conclusion}

Based on findings, this study concludes that the Point of Care, Alere ${ }^{\mathrm{TM}}$ q HIV-1/2-Detect using the AlereTM q platform; has comparative accuracy, sensitivity and specificity with the Standard of Care; COBAS ${ }^{\circledR}$ AmpliPrep/COBAS ${ }^{\circledR} \quad$ TaqMan ${ }^{\circledR} \quad$ HIV-1 Qualitative Test, version 2.0 on the Roche Cobas CAP/CTM 48 or 96 platforms; in the detection of HIV infection in infants. Also, the Point of Care testing for Early Infant Diagnosis has same day result return, is acceptable, rapid and robust; hence, viable and feasible for use in the early infant diagnosis of HIV in Nigeria. However, it is critical to note that there is the need for indepth training and close monitoring when the equipment will be handled by non-laboratorybased personnel.

Society, 21(11), e25209. Date of Access: 15/5/2020 https://doi.org/10.1002/jia2.25209.

[6] Udochisom C. Anaba, Nadia A. Sam-Agudu, Habib O. Ramadhani, Nguavese Torbunde, Alash'le Abimiku, Patrick Dakum, Sani H. Aliyu, Manhattan Charurat, 2019. Missed opportunities for early infant diagnosis of HIV in rural North-Central Nigeria: A cascade analysis from the INSPIRE MoMent study. PLoS ONE. Date of Access: 15/5/2020. https://doi.org/10.1371/journal.pone.0220616.

[7] Isah HO, Ogum E, Cornelius LJ, Okundaye JN, Galadanci H, Charurat ME, et al. 2014. The MoMent Study: Client- and Community-Level Barriers to PMTCT Access and Uptake in Rural North-Central Nigeria. 8th INTEREST (International Workshop on HIV Treatment, Pathogenesis, and Prevention Research in Resource-Poor Settings) Workshop; Lusaka, Zambia. p. 45. Date of Access: 15/5/2020. http://regist2.virology-

education.com/abstractbook/2014_3.pdf.

[8] Leroy, V., Malateste, K., Rabie, H., Lumbiganon, P., Ayaya, S., Dicko, F., Davies, M. A., Kariminia, A., Wools-Kaloustian, K., Aka, E., Phiri, S., Aurpibul, L., Yiannoutsos, C., Signaté-Sy, H., Mofenson, L., Dabis, F., \& International IeDEA Pediatric Working Group1 (2013). Outcomes of antiretroviral therapy in children in Asia and Africa: a comparative analysis of the IeDEA pediatric multiregional collaboration. Journal of acquired immune deficiency syndromes (1999), 62(2), 208- 
219. Date of Access: 14/04/2020 https://doi.org/10.1097/QAI.0b013e31827b70bf.

[9] Hsiao N-y, Dunning L, Kroon M, Myer L. 2016. Laboratory Evaluation of the Alere q Point-of-Care System for Early Infant HIV Diagnosis. PLOS ONE 11(3): e0152672. Date of Access: 5/04/2020 https://doi.org/10.1371/journal.pone.0152672.

[10]Dunning, L., Kroon, M., Hsiao, N. Y., \& Myer, L. 2017. Field evaluation of HIV point-of-care testing for early infant diagnosis in Cape Town, South Africa. PLoS ONE, 12(12), e0189226. Date of Access: 5/04/2020. https://doi.org/10.1371/journal.pone.0189226.

[11] WORLD HEALTH ORGANIZATION (WHO). (WHO). July 2017. Novel Point-of-Care Tools for Early Infant Diagnosis of HIV. Date of Access: 5/04/2020.

https://apps.who.int/iris/bitstream/handle/10665/255

857/WHO-HIV-2017.16-eng.pdf? sequence $=1$.

[12] Peeling R W, Holmes K K, Mabey D, et al. 2006. Rapid tests for sexually transmitted infections (STIs): the way forward Sex Transm Infect 2006 82: v1-v6. doi: 10.1136/sti.2006.024265. Date Accessed $6 / 05 / 2014$.

https://www.who.int/tdr/publications/journalsupplements/sti-way-forward/en/.

[13]Pharr, Jennifer R., Obiefune, Michael C.; Ezeanolue, Chinenye O.; Osuji, Alice; Ogidi, Amaka
G.; Gbadamosi, Semiu, Patel, Dina, Iwelunmor, Juliet; Yang, Wei; Ogedegbe, Gbenga; Ehiri, John E; Sam-Agudu, Nadia A.; Ezeanolue, Echezona E. 2016. Linkage to Care, Early Infant Diagnosis, and Perinatal Transmission Among Infants Born to HIVInfected Nigerian Mothers: Evidence from the Healthy Beginning Initiative, JAIDS Journal of Acquired Immune Deficiency Syndromes: August 1, 2016 - Volume 72 - Issue - p S154-S160. Date of Access: 5/04/2020.

https://doi.org/10.1097/QAI.0000000000001051. [14] Aliyu, M. H., Blevins, M., Megazzini, K. M., Audet, C. M., Dunlap, J., Sodangi, I. S., Gebi, U. I., Shepherd, B. E., Wester, C. W., \& Vermund, S. H. 2014. Correlates of suboptimal entry into early infant diagnosis in rural north central Nigeria. Journal of acquired immune deficiency syndromes (1999), 67(1), e19-e26. Date of Access: 5/04/2020. https://doi.org/10.1097/QAI.0000000000000215.

[15] Simmonds, F.M., Cohn, J.E., Mafaune, H.W. et al. Task shifting for point-of-care early infant diagnosis: a comparison of the quality of testing between nurses and laboratory personnel in Zimbabwe. Hum Resour Health 18, 4 (2020). Date of Access: 05/05/2020 https://doi.org/10.1186/s12960020-0449-2. 